\title{
Sensor-based Mobile Functional Movement Screening
}

\author{
Ulf Jensen $^{1}$, Fabian Weilbrenner ${ }^{1}$, Franz Rott ${ }^{2}$, and Bjoern Eskofier ${ }^{1}$ \\ 1 Pattern Recognition Lab, University of Erlangen-Nuremberg, Germany \\ \{ulf.jensen, bjoern.eskofier\}@cs.fau.de \\ fabian.weilbrenner@informatik.stud.uni-erlangen.de \\ 2 adidas innovation team ait, adidas Group, Herzogenaurach, Germany \\ franz.rott@adidas.com
}

\begin{abstract}
The Functional Movement Screen $^{\mathrm{TM}}$ (FMS) is a useful tool to assess functional abilities in a pre-participation screening. Its seven dynamic movement tests reveal shortcomings in stability and mobility and screen the whole body. However, the current test protocol delivers results that are subjective, qualitative and have to be manually processed. This article presents a semi-automatic system to overcome these limitations for the Deep Squat test. The system consists of four wireless inertial sensors and a central Android ${ }^{\mathrm{TM}}$-based processing node for data analysis and result storage. We developed our system based on data from ten subjects and evaluated the results with the FMS scoring guidelines. The sensor-based scoring system completely agreed with the manual scoring in eight out of ten subjects. In addition, quantitative information in case of compensation movements was logged. Thus, our system is capable of simplifying the FMS test and enhances the score with objective, quantitive and automatic results.
\end{abstract}

Key words: Functional Movement Screen ${ }^{\mathrm{TM}}$, Body Sensor Network, intertial sensors, semi-automatic screening, Android ${ }^{\mathrm{TM}}$ app

\section{Motivation}

Functional movement assessment is established as a valuable procedure to test the preparedness of an individual for activity. It is combined with a prior medical examination and a following performance test for a comprehensive preparticipation screening. Thereby, functional examination reveals shortcomings in mobility and stability during basic functional movements. Seven of these movements have been defined as a standard full-body functional athlete assessment procedure, the Functional Movement Screen ${ }^{\mathrm{TM}}$ (FMS) [1, 2]. The FMS comprises of predefined tests (e.g. special squats and push-ups) and rates individuals according to the quality and efficiency of their execution. Thus, it identifies movement inabilities and is a valuable tool for sports medicine as injuries and irritations can be proactively avoided. Currently, the seven FMS tests are scored by an experienced rater and manually entered into a result sheet. Additional qualitative information that further specifies the score is optional and can be 
added in textual form. This procedure is rater-dependent, based on personal visual assessment and not automated in result processing.

The FMS is widely used in training science and sports medicine and established as a standard tool to assess functional movement abilities. A study that investigated the correlation between core stability, functional movement and performance concluded that the FMS scores quality and efficiency of movement [3]. They further conclude that the FMS is a suitable indicator for risk of injury and is not appropriate to predict performance. The performance has to be assessed in other parts of the pre-participation screening. These findings have been confirmed for sport-specific movements and the authors of [4] proposed to investigate athlete strength instead.

Peate et al. used the FMS to determine the risk of injury for professional firefighters [5]. The authors proved that the number of injuries can be decreased for individuals with a low FMS score with an adequate training program. Thus, they further support the findings that individuals with a lower FMS score are more likely to sustain an injury. The influence of specific training on the results of the FMS test with special emphasis on symmetry were investigated in [6]. The authors proved that the prescribed training helped to reach a FMS score above a predefined threshold score. Asymmetries detected during functional movement assessment were reduced with the training program.

The problem of interrater variability was addressed in [7] and the authors found a good agreement of expert raters and trained novices on videotaped tests. Nevertheless, some FMS tests revealed differences in the scoring and prove the limitations of human assessment. In addition, only a small group of raters was investigated, the intrarater and intrasession variability was not considered and the scoring was made on videotaped tests. Some of these limitations were addressed in [8] that investigated intersession and interrater reliability in real-time testing with the same number of raters. Again, the results proved that novice raters perform also well in real-time FMS scoring. However, the results from the intersession tests revealed a need for reliable scoring of the same person in different sessions as some tests showed only moderate or good reliability.

Body Sensor Networks (BSN) are used for different health and sports applications and are capable of collecting mobile physiological and kinematic data [9]. Software frameworks for the mobile sensor data collection for specific BSN components exist [10]. From an application perspective, BSN are e.g. applied in gait analysis for Parkinson's disease early detection and therapy monitoring [11] and in fatigue classification during activity [12]. Due to their miniaturization and low cost, inertial sensors gain more and more popularity and are applied for numerous purposes in medical engineering, movement science and consumer electronics.

This paper presents a system to address the limitations of the FMS which is a well-established screening test for injury prediction and training control. We propose a semi-automatic system that uses data from inertial body-worn sensors to determine more objective results, to enhance the score with quantitative results and to enable easier result processing. 


\section{Methods}

\subsection{Functional Movement Screen}

The FMS consists of seven dynamic movement patterns to screen the whole body functional movement abilities $[1,2]$. The collected results can be further interpreted by medical or coaching experts for corresponding clinical implications. Thus, scoring results directly pinpoint affected muscle groups or joints that are prone to injury.

Each of the seven tests is scored on a 0-3 ordinal scale. The highest score of 3 is reached if the movement pattern is completed as described in the test specification. If the test is completed with compensational movements a score of 2 is given. Incomplete movement execution results in a score of 1 and the test is scored with 0 if any pain is involved during movement. Specific error patterns and scoring aspects have been defined for each test. Tests are repeated three times and, if the test is of asymmetrical nature, repeated for each side. The lowest score of all repetition of a single test is considered and summed up for an overall score that ranges between 0 and 21 .

Beside the score, the rater can add textual comments that further specify the error pattern, level of compensation or affected side. This information pinpoints shortcomings and is a of great value for therapy planning and monitoring.

\subsection{Overhead Squat}

In our study, we focused on the Deep Squat test [1] that challenges the mechanics of the whole body. In this test (fig. 1), the individual performs a deep squat with an overhead dowel. The Deep Squat is a symmetric test and therefore repeated three times. If the tested individual does not reach the perfect score, the test is repeated with a heel-supporting block. From a functional perspective, the scoring is based on the deepness of the squat, the torso position and knee alignment during testing and the heel movement. We derived four typical error patterns that our systems addressed:

1. The heel is lifted during testing.

2. The femur is not below horizontal in end position.

3. The torso performs a compensational movement in the frontal plane.

4. The torso performs a compensational movement in the sagittal plane.

In addition, it is required that our system automatically detects one repetition of the test to enable the precise error detection and assign detailed information to specific repetitions.

\subsection{Body Sensor Network}

Our BSN data collection system consisted of four SHIMMER ${ }^{\mathrm{TM}}$ sensors [13] and a recording laptop. We positioned the sensor nodes on the dowel, the heel, the shank and the femur as displayed in figure 2. We chose to place the sensors on the right leg and heel. The internal three-axis accelerometer was set to a range of $2 \mathrm{~g}$ and was sampled with a frequency of $100 \mathrm{~Hz}$. Data were wirelessly 

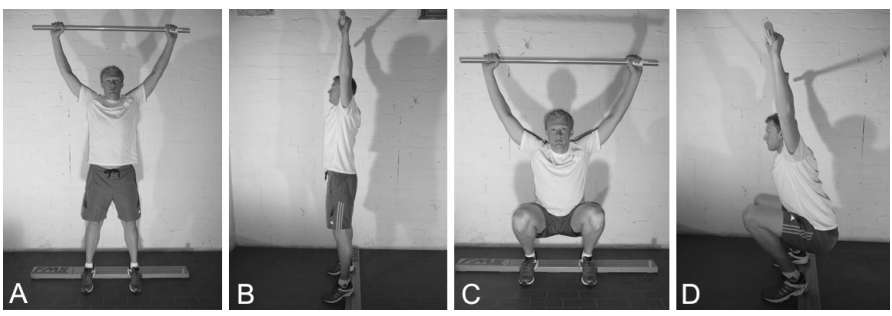

Fig. 1. Overview of the Deep Squat test. Initial positions in frontal view (A) and sagittal view (B) are shown on the left. End positions in frontal view (C) and sagittal view (D) are shown on the right. Positions are shown with the supporting heel block.

transmitted to the recording laptop and stored for further processing. The sensor data were calibrated with the standard procedure specified in the manufacturers user manual.

\subsection{Data Collection}

We collected Deep Squat test data from ten (1 female, 9 male) volunteers and videotaped the experiments for algorithm development and scoring. We used two videocameras for a frontal and a sagittal view of each repetition. As we did not live score the test during data collection, the test was always repeated with a heel-supporting block.

We followed a simple protocol in the test execution. First, the test was explained to the subjects and the sensors were placed. Second, the subjects were put in correct initial position. This position was recorded with the sensors and the videotaping was started. Third, the volunteers were asked to perform three squats. Fourth, the test was repeated with a heel-supporting block.

\subsection{Repetition Detection}

The automatic detection of error patterns required information about the beginning and the end of a repetition during testing. We therefore tracked the subjects' knee angle with data from the sensors on the femur and the shank. It was assumed that the knee behaved like a hinge joint during test execution so that the sensors were only rotated in the sagittal plane.

The knee angle calculation required the initial position $\mathbf{r}_{\mathbf{i}}$ before the test was started and the current position $\mathbf{x}_{\mathbf{i}}$ during repetitions for each sensor $i \in\{1,2\}$ during testing. We calculated the current deviation angle $\alpha_{i}$ with regard to the starting position for each sensor $i$ with eq. 1 .

$$
\alpha_{i}=\arccos \left(\frac{\mathbf{r}_{\mathbf{i}} \cdot \mathbf{x}_{\mathbf{i}}}{\left\|\mathbf{r}_{\mathbf{i}}\right\| \cdot\left\|\mathbf{x}_{\mathbf{i}}\right\|}\right)
$$

The resulting knee angle $\alpha_{\text {knee }}$ was calculated with eq. 2 .

$$
\alpha_{k n e e}=\alpha_{1}+\alpha_{2}
$$

These computations resulted in the knee angle for each sampled sensor value. We averaged the angle results of ten measurements to eliminate outliers and 

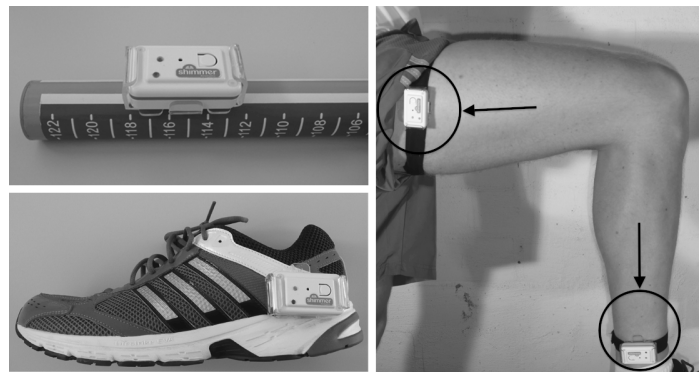

Fig. 2. Overview of sensor placement on the dowel (top left), the heel (bottom left) and the leg (right).

reduce noise. This averaging step was performed for all angle computations.

The knee angle was initialized during the idle phase in correct initial position. We set the value to zero in initial position with extended legs, thus the angle increased with knee flexion.

\subsection{Error Pattern Determination}

Heel Lift According to test specification, the heel has to remain on the floor during test execution. We tracked the heel sensor to detect this error pattern and used eq. 1 for the computation.

Final Femur Position Concerning the final position of the femur we used the same principle. The deviation angle of the femur sensor was calculated with eq. 1. In addition the maximum femur angle was recorded.

Torso Compensation A compensational movement during a deep squat is composed of the compensation in sagittal plane and in frontal plane. In the Deep Squat scoring scheme, both aspects are regarded as one. As the sensor's coordinate system planes roughly coincided with the subjects plane, we were able to discriminate between the frontal and the sagittal plane and quantified the compensation angles with data from the dowel sensor. The difference in the planes were compensated with data from the initial position before test start. Thereby, we projected the three-dimensional vectors $\mathbf{r}_{\mathbf{i}}$ and $\mathbf{x}_{\mathbf{i}}$ in either the frontal or the sagittal plane. As the planes were defined by the axes of the sensor coordinate system, we deleted the corresponding dimension resulting in the twodimensional projections $\hat{\mathbf{r}}_{\mathbf{i}}$ and $\hat{\mathbf{x}}_{\mathbf{i}}$. We used eq. 3 to compute the deviation angle for each plane in two-dimensional space.

$$
\alpha_{i}=\arccos \left(\frac{\hat{\mathbf{r}_{\mathbf{i}}} \cdot \hat{\mathbf{x}}_{\mathbf{i}}}{\| \hat{\mathbf{r}_{\mathbf{i}}\|\cdot\| \hat{\mathbf{x}}_{\mathbf{i}} \|}}\right)
$$

Only compensation movements during repetition were considered. We registered the error if a compensation was detected in at least one of the planes.

\subsection{Implementation}

The algorithms for repetition detection and error pattern determination were implemented in an Android ${ }^{\mathrm{TM}}$ app running on Android-based devices like smart 
phones or tablets. The app built upon the software framework introduced in [10] which manages the connection between the sensors and the analysis device. We set up a user interaction with detailed descriptions of the FMS tests, user and test management and an automatic sensor calibration. The app included a database into which the results were automatically entered after three detected repetitions. Detailed quantified information like the knee angle and the compensation in frontal and sagittal plane were added accordingly.

\subsection{Evaluation}

The videotaped tests were scored and commented by a sport scientist according to the scoring guidelines in [1]. The automatic system scored accordingly and the score was decremented if at least one error pattern was registered. Thus, if one of the error patterns occurred in the first trial without heel-supporting block, the score was decremented. Subsequently, the second trial with heel support was considered and scored in the same way. Finally, the subject was asked if any pain occurred during testing.

To evaluate our sensor-based semi-automatic system, we compared the results from the manual scoring (MANUAL) and our system (SENSOR). For this, we reran the recorded experiments with the Android implementation in simulation mode.

\section{Results}

To determine the beginning and the end of a repetition, we used a threshold value of 20 degrees from the knee angle computation. With this setting, our algorithms was able to correctly detect all squats performed during data collection.

The thresholds for the detection of the error patterns were chosen according to the manual scoring. The final scoring results are compiled in tab. 1.

Table 1. Overview of the results of the manual (MANUAL) and sensor-based (SENSOR) scoring of the Deep Squat test. Results are given for subjects S1 - S10.

\begin{tabular}{c|cccccccccc} 
& S1 & S2 & S3 & S4 & S5 & S6 & S7 & S8 & S9 & S10 \\
\hline MANUAL & 2 & 2 & 1 & 1 & 2 & 1 & 2 & 2 & 2 & 2 \\
SENSOR & 2 & 2 & 1 & 1 & 2 & 2 & 2 & 1 & 2 & 2
\end{tabular}

\section{Discussion}

The results revealed that our system was capable of adequately scoring the Deep Squat test of the FMS. However, the algorithms are based on manually defined thresholds. In future research, these thresholds have to be defined by experienced raters or identified in a larger data collection study. In addition, some error patterns described in the FMS scoring scheme were not covered by the sensorbased system. In the Deep Squat test, this was the case for the alignment of the knee and the foot which has to be addressed in future research.

The Deep Squat test was chosen to provide a proof of concept of a sensor-based FMS scoring system and we are planning to integrate the missing six tests in 
future research. Some of the algorithms like the torso compensation are reusable for other tests with only little modification. However, the use of inertial sensors is not straightforward for tests like the Active Straight Leg Raise test or the Shoulder Mobility test. Nevertheless, a semi-automatic system will be capable of supporting these tests in result entry and storage.

We analyzed the subjects for which the scoring results did not agree in detail. In the test of subject S6, we detected a sagittal compensation movement in the video. However, this compensation was not detected by the sensor system. For subject S8, in contrast, a compensation movement was detected in the sensor result but not visible in the video data. This discrepancy was due to the threshold value we manually set. As mentioned before, the thresholds have to be confirmed by experts or quantitatively computed on a large database. We propose a training phase for our system where error patterns of different severity are scored by FMS experts to set the threshold angles adequately. However, one has to keep in mind that the scoring is subjective and that different raters will use different scales in their personal rating. Thus, this rating has to be repeated and averaged with different raters. The advantage of the resulting averaged automatic system is that it will always compute the same score for one single data set. A human rater, in contrast, might score different results on the same experiment if the scoring is repeated.

Furthermore, the sensor results need to be validated to prove the correctness of the measurement. This is particular important in the case for the final femur position error pattern where a specific angle was defined in the scoring manual. It has to be proven that the sensor systems is capable of computing the correct value. If this is the case, the quantitative sensor output can be interpreted from a biomechanical perspective and enhances the FMS score. We also experienced a couple of factors that interfere in the sensor measurements. These were the initial position of the sensor straps and the movement of sensor during testing due to muscle activity. We are planning to address these issues with the aid of a 3-D motion tracking system to validate the sensor data and quantify the influence of sensor position and movement.

We speculate that quantitative results are helpful for a rater and deduct two application scenarios. First, the support of an unexperienced rater that mainly relies on the automatic scoring and only changes the results if needed. Second, the support of an experienced rater with figures to tackle unclear cases. Beside the scoring aspect, these quantitative results can be used in therapy monitoring to identify improvements that are not reflected in an improvement of the score. An athlete might for example reduce its sagittal compensation by five degrees but still be scored with 1 . Further research has to be conducted to prove the value of quantitative results. It can either be used for assisting the scoring or for a more detailed result than the $0-3$ ordinal scale.

\section{Summary and Outlook}

We presented a sensor-based system to simplify the FMS scoring and to produce more objective and quantitative results. It consisted of four body-worn 
accelerometer sensors and a mobile Android device for data analysis. The comparison of manual and automatic scoring confirmed the applicability of our system to accomplish the scoring of the Deep Squat test.

However, challenges for training the system and sensor placement were identified to further improve our system. In addition, the system needs to be expanded to cover the complete FMS test. We are also planning to enhance our system with data mining functionality to facilitate therapy monitoring and team statistics.

\section{Acknowledgments}

The authors would like to thank all volunteers who participated in the study. This work was funded by the Bavarian Ministry for Economic Affairs, Infrastructure, Transport and Technology and the European Fund for Regional Development.

\section{References}

1. Cook, G. et al.: Pre-Participation Screening: The Use of Fundamental Movements as an Assessment of Function - Part 1. North American Journal of Sports Physical Therapy 1(2), 62-72 (2006)

2. Cook, G. et al.: Pre-Participation Screening: The Use of Fundamental Movements as an Assessment of Function - Part 2. North American Journal of Sports Physical Therapy 1(3), 132-139 (2006)

3. Okada, T. et al.: Relationship between Core Stability, Functional Movement, and Performance. Journal of Strength and Conditioning Research 25(1), 252-261 (2011)

4. Parchmann, C. et al.: Relationship between Functional Movement Screen and Athletic Performance. Journal of Strength and Conditioning Research 25(12), 3378-3384 (2011)

5. Peate, W. et al.: Core strength: A new model for injury prediction and prevention. Journal of Occupational Medicine and Toxicology 2(1), Art. Nr. 3 (2007)

6. Kiesel, K. et al.: Functional Movement Test Scores Improve Following a Standardized Off-season Intervention Program in Professional Football Players. Scand J Med Sci Sports 21(2), 287-292 (2011)

7. Minick, K. et al.: Interrater Reliability of the Functional Movement Screen. Journal of Strength and Conditioning Research 24(2), 479-486 (2010)

8. Onate, J. et al.: Real-time Intersession and Interrater Reliability of the Functional Movement Screen. Journal of Strength and Conditioning Research 26(2), 408-415 (2012)

9. Chen, M. et al.: Body Area Networks: A Survey. Mobile Networks and Applications 2(16), 171-193 (2011)

10. Kugler, P. et al.: Mobile Recording System for Sport Applications. In: Proc. of the 8th International Symposium on Computer Science in Sport (IACSS2011), pp. 67-70, Shanghai (2011)

11. Barth, J. et al.: Biometric and Mobile Gait Analysis for Early Detection and Therapy Monitoring in Parkinson's Disease. In: Proc. of the Annual International Conference of the IEEE Engineering in Medicine and Biology Society (EMBC 2011), pp. 868-871, Boston (2011)

12. Eskofier, B. et al.: Embedded Classification of the Perceived Fatigue State of Runners: Towards a Body Sensor Network for Assessing the Fatigue State during Running. In: Proc. of the 9th International Conference on Wearable and Implantable Body Sensor Networks (BSN 2012), pp. 113-117, London (2012)

13. McGrath, M. and Dishongh, T.: A Common Personal Health Research Platform SHIMMER $^{\mathrm{TM}}$ and BioMOBIUS ${ }^{\mathrm{TM}}$. Intel Technology Journal 13(3), 122-147 (2009) 